\title{
Filtragem direcional radial para a atenuação do ground-roll
}

\author{
Anderson Silva Santos*, Milton José Porsani, INCT-GP/CPGG/IGEO-UFBA, Salvador, BA, Brasil
}

Copyright 2014, SBGf - Sociedade Brasileira de Geofísica.

Este texto foi preparado para a apresentação no VI Simpósio Brasileiro de Geofísica, Porto Alegre, 14 a 16 de outubro de 2014. Seu conteúdo foi revisado pelo Comitê Técnico do VI SimBGf, mas não necessariamente representa a opinião da SBGf ou de seus associados. É proibida a reprodução total ou parcial deste material para propósitos comerciais sem prévia autorização da SBGf.

\section{Resumo}

Apresentamos um novo método de filtragem direcional radial (FDR) de eventos lineares presentes em dados sísmicos de reflexão. A filtragem é realizada em todas amostras de uma família de sismogramas de ponto de tiro comum, através da derivada direcional, tendo um único foco de referência. Para atenuação do groundroll o foco é definido na proximidade da fonte sísmica. O método convencional de derivada direcional efetua a transformada radial (TR), levando os dados do domínio $x$ t para o domínio $x-\theta$ e em seguida é obtida a derivada ao longo na direção $\theta$, após o que é realizada a TR inversa. O método FDR efetua localmente, em cada ponto do grid, a derivada direcional evitando os ruídos numéricos do método TR que ocorrem devidos à reamostragem dos dados. Ilustramos a aplicação do método FDR na filtragem do ground-roll de uma linha sísmica da Bacia do Tacutu. Os resultados numéricos mostram a eficácia da FDR na atenuação daquele tipo de ruído, assim como na melhoria dos espectros de velocidades e na seção empilhada, comparado com o método de filtragem FK.

\section{Introdução}

O processamento sísmico de reflexão visa apresentar uma imagem das estruturas geológicas em subsuperfície o mais próxima da realidade possível. Mas isso constitui um sério desafio para o geofísico, pois os dados obtidos durante a aquisição apresentam um conjunto de eventos indesejáveis que reduzem bastante a razão sinal/ruído na seção sísmica empilhada. Um evento que reduz essa razão é o ruído coerente ground-roll que aparece no sismograma de levantamentos terrestres como um cone de baixa frequências e altas amplitudes (vide Fig. 1).

O ruído coerente ground-roll possui como características, baixas frequências e altas amplitudes, o que prejudica seriamente a razão sinal/ruído no dado de sísmica de reflexão. Por ser um evento bastante energético, acaba mascarando os eventos de interesse no sismograma, prejudicando a análise de velocidades e a qualidade da seção sísmica empilhada.

Nas últimas décadas a indústria do petróleo, em conjunto com a academia, não tem poupado esforços para desenvolver métodos de melhoria da razão sinal/ruído dos sismogramas (Yilmaz, 2001). Embora existam técnicas de configuração de arranjos de geofones que permitam atenuar o ground-roll durante a aquisição, muitas vezes essas são impraticáveis pois encarecem o custo da aquisição ou mesmo não podem ser utilizadas quando os dados já foram adquiridos com métodos convencionais.

Os métodos de filtragem no domínio da frequência (1D ou 2D) atuam de forma cirúrgica e removem também frequências da banda do sinal sísmico, eliminando parte do sinal junto com o ruído que se deseja eliminar, não gerando ganhos significativos na razão sinal/ruído (Manenti et al, 2013; Melo et al. 2008). Um filtro popularmente utilizado para a filtragem de eventos lineares é o filtro FK (Frequência-Número de onda). Neste filtro está envolvida a eliminação de parte de bandas de energia dos eventos no domínio f-k ( frequência-número de onda).

Nesse contexto apresentamos um novo método de filtragem que é a FDR (Filtragem Direcional Radial). Este filtro leva em conta a natureza linear do ruído que se deseja atenuar. Consiste em se tomar um foco no domínio do tiro e a partir desse se calcular as derivadas na direção de todas as amostras do grid, ou seja cada amostra "vê"o foco (ponto de tiro) com um ângulo específico (Santos e Porsani, 2013).

Aplicamos o método em dados sísmicos terrestres da Bacia do Tacutu. Os resultados obtidos, quando comparados com o clássico método de filtragem FK, demonstram que o método FDR é bastante eficiente na atenuação do ground-roll e coloca em evidência reflexões que antes se encontravam mascaradas pela energia do ruído.

\section{A filtragem FK}

A filtragem f-k (Frequência-número de onda) é uma importante ferramenta na etapa de filtragem de dados sísmicos de reflexão, visto que esta usa também informações acerca do número de onda (número de ciclos no espaço) para fazer distinção entre o que é desejável e indesejável no sismograma.

A Transformada de Fourier 2D é utilizada para levar os dados sísmicos do domínio t-x para o domínio f-k, sendo esta expressa através da seguinte equação:

$$
\bar{A}\left(w, k_{x}\right)=\int_{-\infty}^{+\infty} \int_{-\infty}^{+\infty} A(t, x) e^{i k_{x}-i w} d t d x
$$

Sendo $\bar{A}\left(w, k_{x}\right)$ a transformada 2D de Fourier. Onde a primeira transformada de Fourier converte o eixo do tempo para o eixo da frequência e a segunda converte o eixo do espaço para o eixo que representa o número de onda. Sabe-se que os eventos que aparecem em um sismograma possuem diferentes inclinações e uma característica importante ao se mudar do domínio do tx (tempo-espaço) para o domínio f-k (frequência-número de onda) é que esses eventos mudam de inclinação. Os eventos mais inclinados em t-x aparecerão com menor 
inclinação em f-k e a reciproca é verdadeira; já as reflexões que são representadas aproximadamente por hipérboles em $\mathrm{t}-\mathrm{x}$ aparecem de forma vertical em $\mathrm{f}-\mathrm{k}$. Sabendo disse pode-se reservar regiões no domínio f-k que serão eliminadas durante a etapa de filtragem, e logo após realizado 0 processo pode-se retornar 0 dado para 0 domínio $\mathrm{t}-\mathrm{x}$ através da transformada inversa 2D de Fourier.

O filtro FK apresenta uma desvantagem quando o objetivo é preservar a bandas de frequências do sinal durante a etapa de filtragem, pois ao se definir uma zona de rejeição no domínio f-k, uma parte do sinal também é rejeitada.

\section{A Filtragem Direcional Radial}

O método de filtragem FDR consiste em se tomar um foco no domínio do tiro e tomando esse como referência se calcula a derivada na direção de todas as amostras do grid. As derivadas são calculadas utilizando operadores de derivadas 2D que são obtidos através de uma aproximação numérica para as derivadas por meio de diferenças finítas (Mello et al, 2008).

O objetivo desses operadores de derivadas é estimar a derivada em uma região na matriz dos dados que podemos tomar como coordenadas $\mathbf{A}(n z, n x)$, onde $n z$ representa o número de amostras em um traço e $n x$ representa a quantidade de traços que serão filtrados.

Seja $f(x, t)=f(\vec{r})$ à representação de um campo, onde os valores interpolados em uma direção $\vec{r}$ serão:

$$
\begin{aligned}
& f\left(\vec{r}^{+}\right) \approx \sum_{i=1}^{n} w_{i}^{+} A_{i}=I\left(\vec{r}^{+}\right)=I^{+} \\
& f\left(\vec{r}^{-}\right) \approx \sum_{i=1}^{n} w_{i}^{-} A_{i}=I\left(\vec{r}^{-}\right)=I^{-}
\end{aligned}
$$

No cálculo dos pesos $w_{i}$ usou-se o método de interpolação de Shepard (Shepard, 1968), que é baseada no inverso da distância.

$$
w_{i}=\frac{\frac{1}{d} i}{\sum_{i=1}^{n} \frac{1}{d_{j}}}
$$

onde $d_{j}=\left|\vec{r}-\vec{r}_{i}\right|$.

Portanto a derivada na direção de $\vec{r}$ pode ser estimada através da equação:

$$
\frac{d f(x, t)}{d \vec{r}} \approx \frac{I\left(\vec{r}^{+}\right)-I\left(\vec{r}^{-}\right)}{2 \delta_{r}}=\sum_{i=1}^{n} \frac{\left(w^{+}-w^{-}\right) A_{i}}{2 \delta_{r}},
$$

Onde $\delta_{r}>0$ representa a perturbação na direção $\vec{r}$ Sendo assim o operador de derivada é dado por:

$$
D_{r}=O^{+}-O^{-}=\frac{\left(w^{+}-w^{-}\right)}{2 \delta_{r}}, i=1, \ldots, n .
$$

Se calcular as derivadas irradiando de um foco no domínio do tiro fora pensado com o objetivo de se evitar a filtragem em outros domínios, visto que muitas vezes são gerados ruídos numéricos ao se trabalhar nesses domínios. A escolha do foco foi estudada cabalmente e observouse que a posição que produzia os melhores resultados era quando tomada do ponto de origem do ruído, sendo que quando calculada a derivada na direção do evento inclinado e em seguida aplicada as amostras do grid na respectiva inclinação, o resultado é a atenuação do evento linear presente no sismograma. Como as inclinações dos eventos no sismograma estão relacionados com a velocidade desses, pode-se dizer que assim como o filtro FK, o filtro FDR também é um filtro de velocidades, que no presente trabalho visa atenuar o ruído de baixa velocidade, o ground-roll.

\section{Processamento dos dados utilizando a GêBr}

Com o objetivo de testarmos o novo método de filtragem foi realizado o processamento de uma linha sísmica da Bacia do Tacutu. Dado que fora adquirido pelo CPGGUFBA junto a ANP (Silva e Porsani, 2006). Os parâmetros de aquisição estão apresentados na tabela abaixo.

\begin{tabular}{|c|c|}
\hline \multicolumn{2}{|c|}{ Parâmetros de aquisição } \\
\hline Linha & 204-RL-247 \\
Lanço & 3850-100-0-100-1050 \\
$\mathrm{t}(\mathrm{s})$ & 4 \\
$\Delta t(m s)$ & 4 \\
$\mathrm{GC}(\%)$ & 4800 \\
$\Delta s(m)$ & 50 \\
$\Delta g(m)$ & 50 \\
$\mathrm{Nc}$ & 96 \\
\hline
\end{tabular}

Optou-se por usar a plataforma GêBr de processamento sísmico, de uso público e disponível (Biloti, 2013). O objetivo é mostrar que esta plataforma representa uma poderosa interface para o ensino e pesquisa. A GêBr é uma interface amigável que tem como objetivo fornecer ao usuário a facilidade de processamento que os softwares comerciais apresentam. É construída com base no pacote livre de processamento sísmico, Seismic Unix(SU) do Center for wave Phenomena (CWP) da School of Mines. O SU assim como a plataforma GêBr possuem livre distribuição. Esta plataforma permite a inserção de módulos externos que pode ser desde um programa que realiza a filtragem até ao que realize a migração do dado sísmico com um novo método desenvolvido na acadêmia e na indústria, o que faz dessa plataforma uma excelente oportunidade de mostrar o que é desenvolvido nas instituições de ensino.

\section{Resultados}

Separou-se um shot-gather (Figura 1) que possui o ruído bem evidente assim como reflexões, isto para se observar os efeitos produzidos pela filtragem dos dados.

O resultado da filtragem FK (Figura 2) é bem satisfatório, pois houve uma atenuação significativa do ground-roll, além de revelar as reflexões que antes se encontravam mascaradas, com destaque para a preservação das baixas frequências.

O resultado da filtragem FDR é mostrado na Figura 3 . Percebe-se que a filtragem direcional atenua o groundroll e por atenuá-lo levando em conta a sua linearidade no domínio do tiro, interfere pouco com as reflexões que são de natureza hiperbólica, logo, o resultado é um aumento significativo na razão sinal/ruído e a boa preservação das reflexões hiperbólicas, da região do ground-roll.

A Figura 4 mostra o espectro FK do sismograma original (Fig. 1) e o espectro do dado filtrado no domínio FK. Notar que após a retirada dos eventos "indesejáveis", ocorre um enriquecimento relativo de energia na região central 
do espectro FK. Grande parte da energia presente nessa região pertence as reflexões.

Observando o espectro de amplitude gerado após a filtragem FDR (Figura 5), nota-se uma atenuação moderada na região que se encontrava o ground-roll, o que é extremamente promissor, visto que ocorre uma maior preservação de sinal nessa faixa, possibilitando um aumento da amplitude na faixa do sinal entre $20 \mathrm{~Hz}$ e 30 $\mathrm{Hz}$.

\section{Análise de velocidades}

$\mathrm{Na}$ etapa de análise de velocidades é de fundamental importância que os eventos de interesse possuam a maior coerência possível, visto que no cálculo da semblance os ruídos de natureza coerente podem prejudicar o mapeamento das velocidades.

Como na linha sísmica da Bacia do Tacutu a aquisição foi feita com baixa cobertura, utilizamos super gathers para aumentar a cobertura CDP para melhorar a análise de velocidades. Os super gathers foram gerados com o auxílio de uma shell script.

Percebe-se que abaixo de 2 s o super gather do dado sem filtros (Figura 6) apresenta uma alta contaminação de ruído de alta frequência que está presente em todo dado da linha 204-RL-247. A filtragem FK apesar de melhorar a coerência das medidas de semblance no espectro de velocidades, não atua com eficiência nas altas frequências, visto que o filtro foi usado para atenuar as baixas frequências, região que se encontrava o ruído (Figura 7).

Uma rápida análise da Figura 8 mostra o quanto o método FDR foi eficiente em atenuar o ground-roll e em revelar eventos que se encontravam mascarados pela energia do mesmo. O filtragem através do método FDR conseguiu adicionalmente eliminar o ruído de alta frequência que prejudicava a visualização dos eventos abaixo de $2 \mathrm{~s}$, isso porque esse ruído que se apresentava de forma coerente e vertical em cada receptor fora atenuada durante a aplicação do filtro, que o considerou como um evento de muito baixa velocidade. O resultado é um painel de semblance com maior resolução, o que proporciona um melhor mapeamento das velocidades que serão usadas para correção NMO (Normal Move Out) e no empilhamento da linha sísmica.

\section{Seções empilhadas}

A seção original sem filtros (Figura 9) apresenta uma alta contaminação proveniente do ruído coerente ground-roll, o que dificulta o mapeamento dos refletores nas regiões acima de $1 \mathrm{~s}$. Isso é resolvido após a filtragem FK (Figura 10), onde agora se pode mapear refletores na seção acima de $1 \mathrm{~s}$ e esta agora apresenta uma maior resolução temporal.

O resultado obtido com a seção empilhada após a filtragem FDR (Figura 11) são bastante satisfatórios, percebe-se um aumento significativo da resolução temporal e os refletores mais rasos podem ser melhores mapeados após a filtragem.

\section{Conclusões}

Apresentamos um novo método para filtragem de eventos sísmicos lineares presentes em dados sísmicos de reflexão. No método FDR a filtragem é realizada localmente, em cada ponto da matriz de dados, e os resultados não estão influenciados por erros numéricos devidos à interpolação ao longo das direções radiais, conforme ocorre no método de transformada radial (TR). A implementação computacional do método FDR é relativamente simples e seu custo computacional é baixo, praticamente igual aos métodos de filtragem convencionais, a exemplo da deconvolução. Os resultados numéricos apresentados na filtragem do ruído ground-roll, bem como as melhorias obtidas no espectro de velocidades e na seção sísmica empilhada, comparado aos resultados do método $\mathrm{FK}$, demonstram sua aplicabilidade e benefícios quando da aplicação no processamento de dados sísmicos terrestres.

\section{Agradecimentos}

Os autores agradecem ao CPGG/UFBA/INCT-GP, FINEP, ANP e PETROBRAS pelo apoio no desenvolvimento deste trabalho. Também agradecemos à empresa Landmark pela cessão das licenças dos softwares de processamento de dados sísmicos. Anderson Silva Santos agradece ao CNPq pelo suporte financeiro através da bolsa de pesquisa.

\section{Referências}

Biloti, R. (2013). A plataforma livre de processamento sísmico, GêBR. http://www.gebrproject.com

Manenti, R.R., Porsani, M.J. (2013). Ground Roll Attenuation Applying Adaptive SVD Method in the Radial Domain, Society of Exploration Geophysicists.

Melo, P.E.M., M.J. Porsani e Silva, M. G. (2008). Ground-roll attenuation using a 2D time-derivative filter. Geophysical Prospecting, 2008, 57, 343-353.

Romanelli, A. (2010). Análise do sinal sísmico, Sociedade Brasileira de Geofísica (SBGf). Rio de Janeiro.

Santos, A., Porsani, M.J. (2013). Derivada direcional radial aplicada a filtragem do ground-roll. 3 Workshop da Rede Cooperativa de Pesquisa, Inovação e Transferência de Tecnologia NNE/FINEP de Geofísica de Exploração, Salvador.

Shepard, Donald (1968). A two-dimensional interpolation function for irregularly-spaced data. Proceedings of the 1968 ACM National Conference. pp. $517 a ̂ 524$. doi: $10.1145 / 800186.810616$

Silva, M.G., Porsani, M.J. (2006). Aplicação de Balanceamento espectral e DMO no processamento sísmico da Bacia do Tacutu.Brazilian Journal of Geophysics, Vol. 24(2), 2006.

Yilmaz, O. (2001). Seismic Data Analysis: Processing, Inversion and Interpretation of Seismic Data, Society of Exploration Geophysicists, Tulsa. 


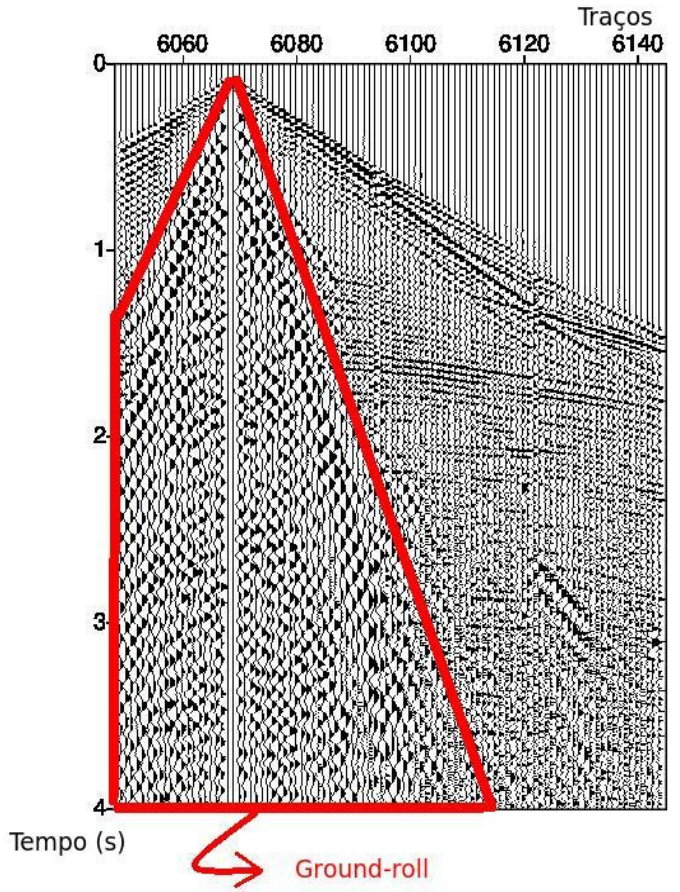

Figura 1: Família de traços de ponto de tiro comum, contaminado com ground-roll.

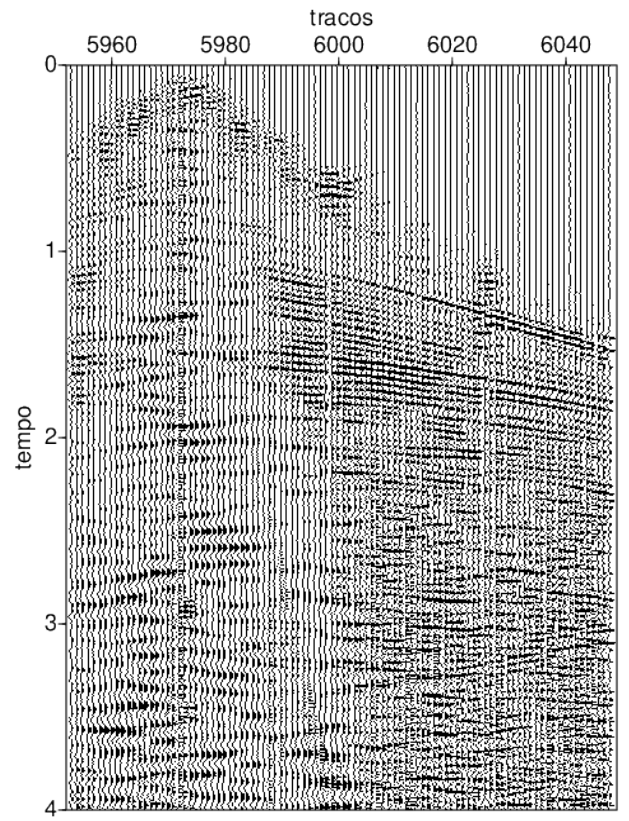

Figura 2: Tiro após a filtragem FK.

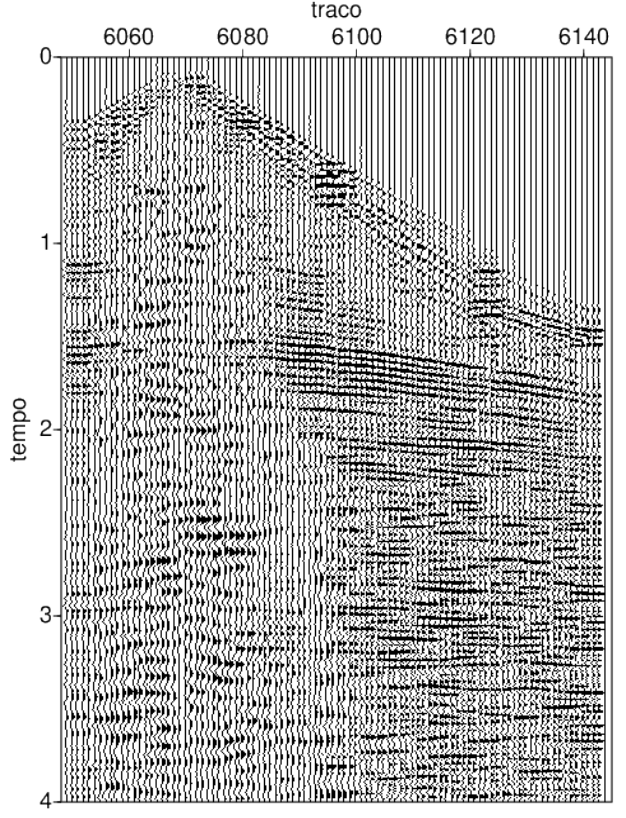

Figura 3: Tiro após a filtragem FDR.

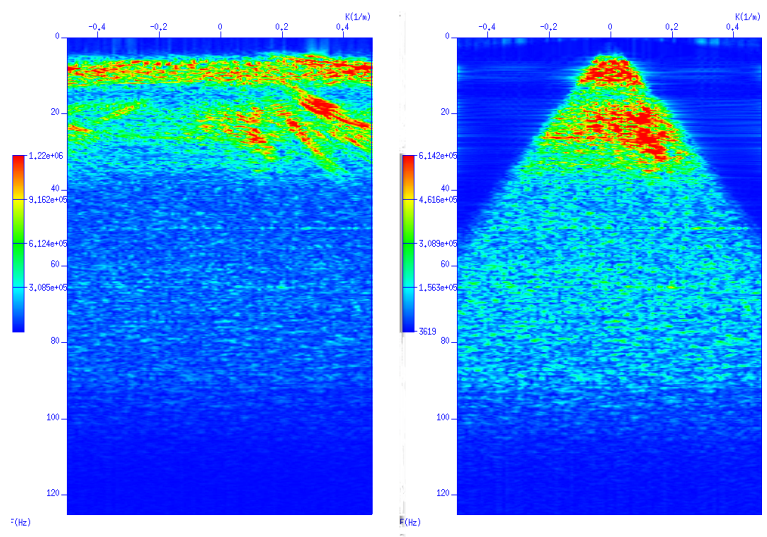

Figura 4: Espectro FK antes e após a filtragem.

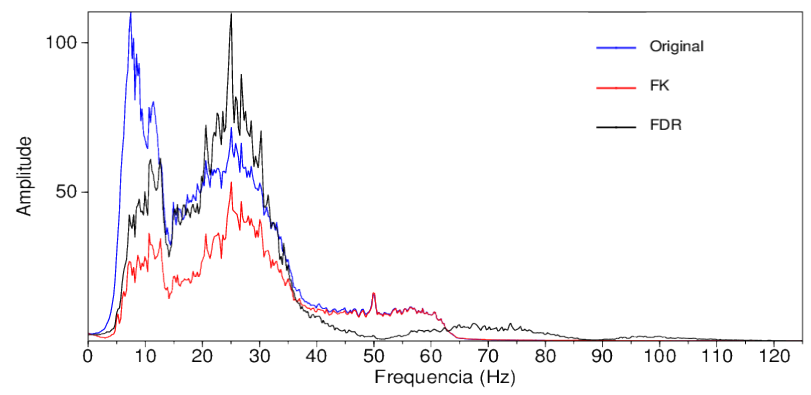

Figura 5: Espectros de amplitudes comparando o efeito das filtragens FK e FDR. 


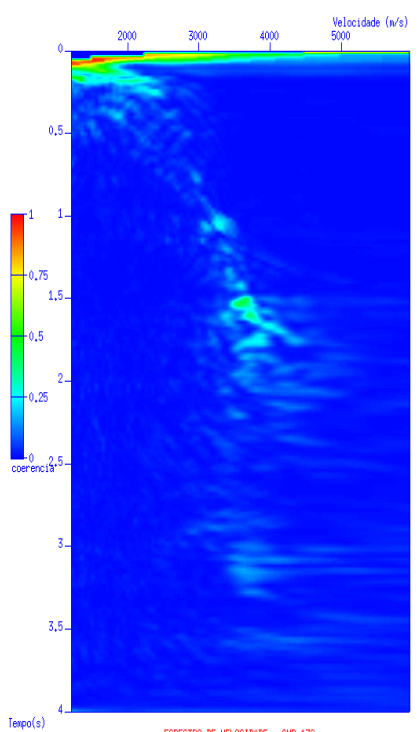

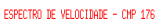

Figura 6: Espectro de velocidades do CMP 176 sem filtros.
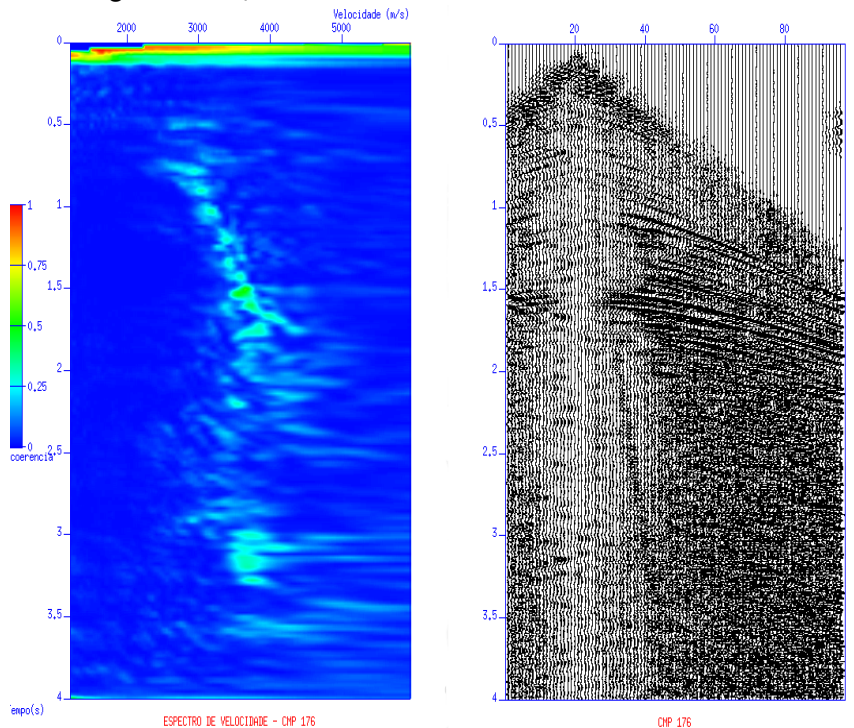

Figura 7: Espectro de velocidades do CMP 176 após a filtragem FK.
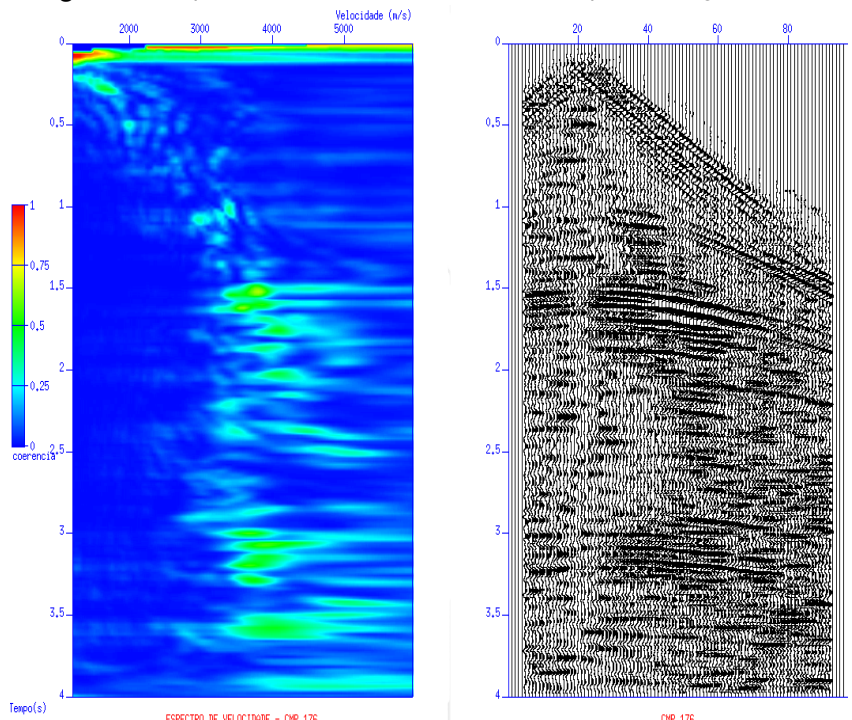

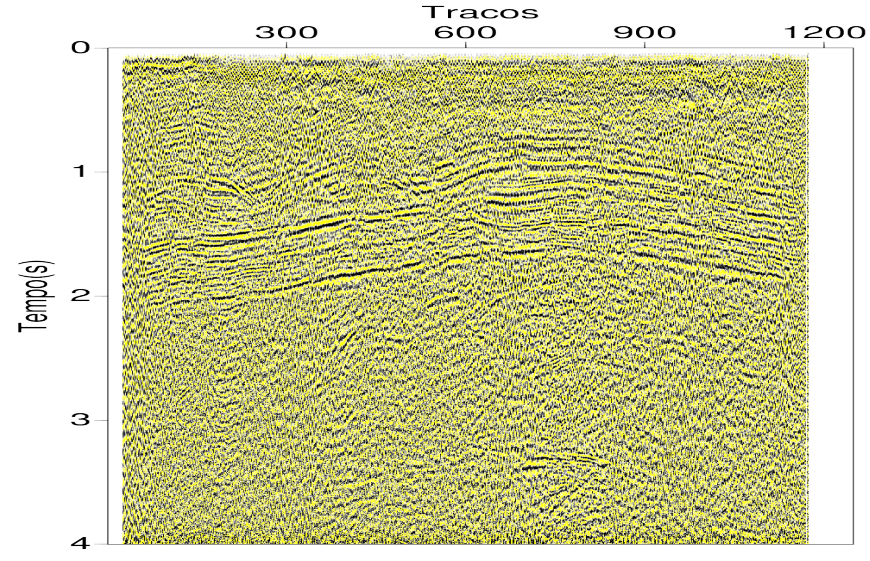

Figura 9: Seção empilhada original.

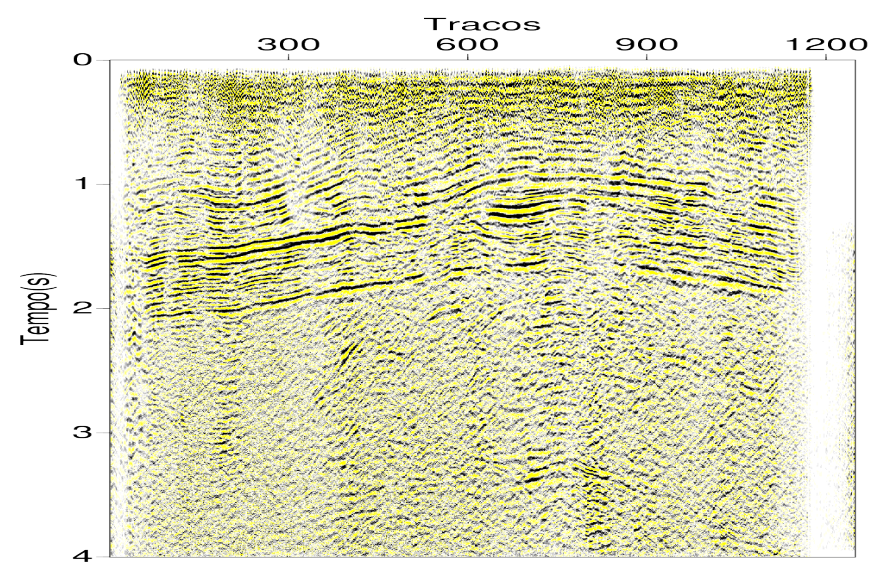

Figura 10: Seção empilhada após o filtro FK.

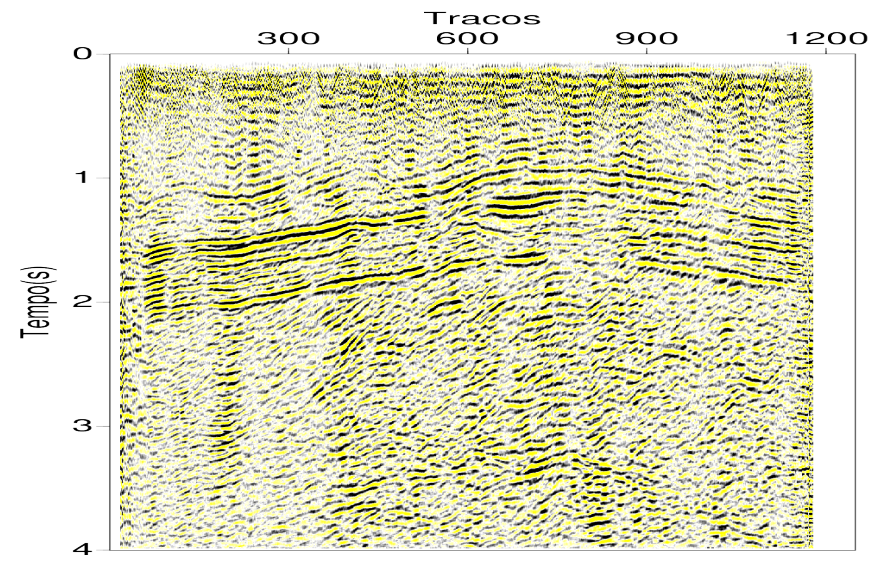

Figura 11: Seção empilhada após o filtragem FDR.

Figura 8: Espectro de velocidades do CMP 176 após a filtragem FDR. 